\title{
Estudos de Modelos Dispersivos para Dinâmica de Populações
}

\author{
William M. S. Yamashita ${ }^{1}$, Lucy T. Takahashi ${ }^{2}$, Grigori Chapiro ${ }^{2}$ \\ ${ }^{1}$ Modelagem Computacional, ${ }^{2}$ Departamento de Matemática, UFJF, \\ 36036-330, Campus Universitário, Universidade Federal de Juiz de Fora, Juiz de Fora, MG \\ E-mail: wmsyamashita@hotmail.com, ltiemi@gmail.com, grigori@ice.ufjf.br.
}

\begin{abstract}
Resumo: Nas últimas décadas, a incidência global da dengue tem crescido dramaticamente favorecida pelo aumento da mobilidade humana e da urbanização. O estudo da população do mosquito é de grande importância para a saúde pública em países como o Brasil, onde as condições climáticas e ambientais são favoráveis para a propagação desta doença. Este trabalho baseia-se no estudo de modelos matemáticos que tratam do ciclo de vida do mosquito da dengue usando equações diferencias parciais. Estudamos a existência de solução na forma de onda viajante, usando um método semi-analítico combinando técnicas de Sistemas Dinâmicos, como a seção de Poincaré e análise local com base no Teorema de Hartman-Grobman, e integração numérica.
\end{abstract}

Palavras-chave: Equações Diferenciais Parciais, Onda Viajante, Leis de Conservação, Dengue

Problema: Atualmente, mais de 2,5 bilhões de pessoas no mundo vivem em risco da dengue, não só nas regiões tropicais, mas também, atingindo climas temperados. O Aedes aegypti se prolifera em estreita proximidade com as comunidades humanas usando armazenamentos artificiais de água tais como tanques, tambores, baldes, vasos de flores, etc., como locais de reprodução [2].

No sentido de epidemias, Hay [3] relata uma possível epidemia de dengue no Brasil em 2014 que sediará a $20^{\circ}$ Copa do Mundo FIFA de futebol. Ele avalia os potenciais níveis de exposição através da análise de mapas de distribuição da dengue no Brasil e os registros de sua variação sazonal nas cidades-sede.

Existem várias abordagens sobre a invasão e busca de controle da dengue. O trabalho de Dufourd e Dumont [1] tem por objetivo desenvolver um modelo matemático para simular a dispersão do mosquito Aedes albopictus e seu controle levando em conta os parâmetros ambientais (vento, temperatura, localização). Os autores usam ferramentas de controle biológico, como a Técnica do Inseto Estéril que consiste na introdução de um grande número de insetos estéreis. Eles fazem simulações numéricas para a dispersão do mosquito e testam diferentes cenários para o controle da doença.

Uma solução de uma equação diferencial parcial (EDP) que pode ser escrita na forma $u(\xi)$ é chamada solução na forma de uma onda viajante, onde a variável $\xi=x-c t$ é dita variável viajante e $c$ (constante) é a velocidade de propagação. Utilizaremos este tipo de solução neste trabalho, que transforma uma EDP em uma equação diferencial ordinária (EDO). Assim, encontrar a solução da EDO na forma de uma onda viajante equivale a encontrar a solução da EDP correspondente.

Com esta forma de solução, destacamos o trabalho de Maidana e Yang [5] que determinam a existência de soluções em forma de ondas viajantes, em processos de invasão do mosquito Aedes aegypti. Os autores propuseram um modelo matemático baseado na difusão espacial da dengue por meio de um sistema de EDP de Reação e Difusão, relacionando as populações humanas e de mosquitos. A taxa de disseminação da doença é determinada pela aplicação das soluções de ondas viajantes para o sistema correspondente de EDP.

Formulação do problema: Passamos agora, para a descrição do ciclo de vida do mosquito da dengue. Focando nossa atenção numa escala espacial urbana onde um processo (local) de difusão 
é devido a um movimento de busca autônomo e aleatório da fêmea alada do Aedes aegypti e é acoplado com uma constante de advecção que pode ser interpretada como um resultado de transporte pelo vento. Faremos nossa análise para o caso unidimensional [9].

Para simplificar a dinâmica biológica vital do mosquito, o modelo proposto em [9] considera somente duas subpopulações, a forma alada e móvel (mosquitos adultos fêmeas) e uma população aquática e estática, na qual incluem as formas ovo, larva e pupa. Considere o seguinte esquema compartimental baseado no processo de desenvolvimento do mosquito

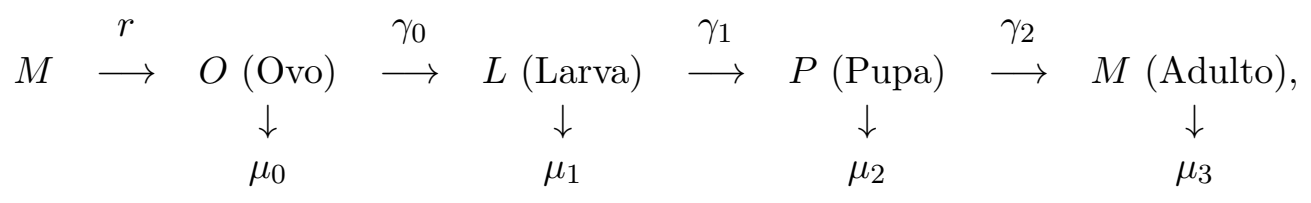

onde $O(x, t), L(x, t), P(x, t)$ e $M(x, t)$ são as densidades de ovos, larvas, pupas e mosquitos fêmeas aladas, respectivamente. Em cada uma destas fases existe uma taxa $\mu_{i}, i=0,1,2,3$, de mortalidade, uma taxa $\gamma_{i}$ de passagem da fase $i$ para a fase $i+1, i=0,1,2$, e uma taxa $r$ de oviposição. Consideramos que o Aedes aegypti possui duas fases: ovo, larva e pupa compõem a fase aquática e estática $(\bar{A}(x, t))$, e mosquitos fêmeas a sua fase alada e móvel $(\bar{M}(x, t))$.

Começando com um modelo simples, os autores de [9] descrevem um modelo para a dinâmica espacial da população de Aedes aegypti. Assim, tem-se

- $\overline{\mu_{i}}$ - taxa de mortalidade ( $i=1$ da fase alada e $i=2$ da fase aquática);

- $\overline{k_{i}}$ - é a capacidade suporte de cada fase;

- $\bar{\gamma}$ - taxa de maturação da forma aquática para a forma alada dos mosquitos fêmeas;

- $\bar{r}$ - taxa de oviposição pelos mosquitos fêmeas;

- $\bar{\gamma} \bar{A}(x, t)\left(1-\frac{\bar{M}(x, t)}{\overline{k_{1}}}\right)$ - termo de Verhulst o qual descreve uma capacidade suporte $\overline{k_{1}}$ relacionada a quantidade de sangue disponível;

- $\bar{r} \bar{M}(x, t)\left(1-\frac{\bar{A}(x, t)}{\overline{k_{2}}}\right)$ - taxa de oviposição é proporcional a sua densidade que é regulada pelo efeito da capacidade suporte sobre a ocupação dos criadouros viáveis.

Considera-se que a dispersão do mosquito da dengue como sendo um resultado de um movimento aleatório (e local) do vôo, representado macroscopicamente pelo processo de difusão $\bar{D}$, acoplado a uma advecção causada pelo vento e um fluxo com velocidade constante $\bar{\nu}$. Em [9] propõem-se o seguinte modelo

$$
\left\{\begin{aligned}
\bar{M}_{t} & =\bar{D} \bar{M}_{x x}-(\bar{\nu} \bar{M})_{x}+\bar{\gamma} \bar{A}\left(1-\frac{\bar{M}}{\overline{k_{1}}}\right)-\overline{\mu_{1}} \bar{M} \\
\bar{A}_{t} & =\bar{r}\left(1-\frac{\bar{A}}{\overline{k_{2}}}\right) \bar{M}-\left(\overline{\mu_{2}}+\bar{\gamma}\right) \bar{A}
\end{aligned}\right.
$$

Após introduzir a escala apropriada, adimensionaliza-se o sistema acima e obtém-se

$$
\left\{\begin{aligned}
M_{t} & =M_{x x}-\nu M_{x}+\frac{\gamma}{k} A(1-M)-\mu_{1} M \\
A_{t} & =k(1-A) M-\left(\mu_{2}+\gamma\right) A,
\end{aligned}\right.
$$

onde $M$ e $A$ são as densidades adimensionalizadas da população alada e da população aquática, respectivamente, e $\nu, \gamma, k, \mu_{1}, \mu_{2}$ são os parâmetros adimensionais.

Após descrever o modelo, os autores de [9] buscam formas para encontrar soluções para o problema, levando em conta o sentido biológico da solução. Como não foi possível encontrar a solução clássica, eles analisaram um exemplo numérico. Estudam a solução em forma de ondas viajantes, ver Figura 1. Buscam a solução das EDO pelo método de Runge-Kutta de quarta ordem e validam essa solução resolvendo numericamente as EDP, usando o método de Crank-Nicolson. 


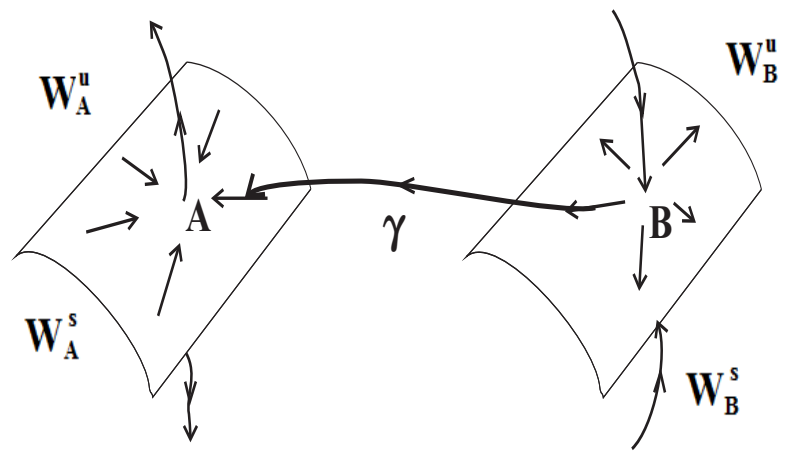

Figura 1: Órbita heteroclínica $(\gamma)$, onde $W_{i}^{s}$ e $W_{i}^{u}$ são as variedades estável e instável, respectivamente, em $A$ e $B$.

Em [2], o modelo acima é generalizado. Os autores modificam as equações de [9] para ter uma melhor descrição dos fenômenos de difusão e advecção. Uma possível justificativa seria o quanto a nuvem de mosquistos está influenciando na propagação da epidemia. Nós iremos trabalhar com o modelo de [2] modificado. Utilizando a mesma notação que no Modelo (3), temos que

$$
\left\{\begin{array}{l}
M_{t}=M_{x x}-2 \nu M^{q} M_{x}+\frac{\gamma}{k} A(1-M)-\mu_{1} M \\
A_{t}=k(1-A) M-\left(\mu_{2}+\gamma\right) A
\end{array}\right.
$$

onde $q>0$ e os parâmetros são os mesmos do Modelo (3).

Método: Os trabalhos citados carecem de detalhes na demonstração da existência de solução, este será o nosso objetivo neste trabalho. Estudaremos soluções na forma de ondas viajantes no Sistema (4). As soluções em forma de ondas viajantes podem ser escritas como

$$
M(x, t)=m(\xi) \quad \text { e } \quad A(x, t)=a(\xi)
$$

onde $\xi=x-c t$, $c$ é uma velocidade constante, $m(\xi)$ e $a(\xi)$ são os perfis de onda. Olhamos para os perfis que levam à interpretação de uma invasão, tais que

$$
\left\{\begin{array}{lll}
\lim _{\xi \rightarrow-\infty} m(\xi)=m^{-} & \text {e } & \lim _{\xi \rightarrow+\infty} m(\xi)=0, \\
\lim _{\xi \rightarrow-\infty} a(\xi)=a^{-} & \text {e } & \lim _{\xi \rightarrow+\infty} a(\xi)=0,
\end{array}\right.
$$

onde $m^{-}$e $a^{-}$são os limites das populações.

De (5), temos que

$$
M_{t}=-c m^{\prime}(\xi), M_{x}=m^{\prime}(\xi), M_{x x}=m^{\prime \prime}(\xi) \text { e } A_{t}=-c a^{\prime}(\xi),
$$

onde $m^{\prime}=d m / d \xi$ e $a^{\prime}=d a / d \xi$. Substituindo (7) no Sistema (4) e reescrevendo o sistema na forma de um sistema de $1^{\mathrm{a}}$ ordem, obtemos

$$
\left\{\begin{aligned}
m^{\prime}(\xi) & =h(\xi) \\
h^{\prime}(\xi) & =\left(2 \nu m^{q}(\xi)-c\right) h(\xi)+\left(\mu_{1}+\frac{\gamma}{k} a(\xi)\right) m(\xi)-\frac{\gamma}{k} a(\xi) \\
a^{\prime}(\xi) & =\frac{k}{c}(a(\xi)-1) m(\xi)+\left(\frac{\mu_{2}+\gamma}{c}\right) a(\xi),
\end{aligned}\right.
$$

com as equivalentes condições de contorno de (6)

$$
\left\{\begin{array}{lll}
\lim _{\xi \rightarrow-\infty} m(\xi)=m^{-} & \text {e } & \lim _{\xi \rightarrow+\infty} m(\xi)=0 \\
\lim _{\xi \rightarrow-\infty} h(\xi)=0 & \text { e } \lim _{\xi \rightarrow+\infty} h(\xi)=0 \\
\lim _{\xi \rightarrow-\infty} a(\xi)=a^{-} & \text {e } \lim _{\xi \rightarrow+\infty} a(\xi)=0 .
\end{array}\right.
$$


Buscamos os pontos singulares do Sistema (8), que são obtidos resolvendo o sistema homogêneo $\left(m^{\prime}, h^{\prime}, a^{\prime}\right)=(0,0,0)$. Neste sistema, tomando o limite quando $\xi \rightarrow-\infty$ e usando $(9)$, obtemos

$$
\left\{\begin{array}{l}
\gamma\left(m^{-}-1\right) a^{-}+k \mu_{1} m^{-}=0 \\
k\left(a^{-}-1\right) m^{-}+\left(\mu_{2}+\gamma\right) a^{-}=0 .
\end{array}\right.
$$

Resolvendo este sistema, obtemos os pontos singulares $A=(0,0,0)$ e $B=\left(m^{-}, 0, a^{-}\right)$, onde

$$
a^{-}=\frac{k\left(\gamma-\mu_{1} \mu_{2}-\gamma \mu_{1}\right)}{\gamma\left(k+\mu_{2}+\gamma\right)} \quad \text { e } \quad m^{-}=\frac{\gamma-\mu_{1}\left(\mu_{2}+\gamma\right)}{\gamma+k \mu_{1}} .
$$

Estudamos a estabilidade do fluxo do Sistema (8) na vizinhança dos equilíbrios, com base no Teorema Hartman-Grobman [7]. Consideramos $F(m, h, a)=m^{\prime}, G(m, h, a)=h^{\prime}$ e $H(m, h, a)=$ $a^{\prime}$ e calculamos a matriz Jacobiana $(J)$ do Fluxo (8). Assim, para o ponto singular $A=(0,0,0)$, obtemos

$$
J(A)=J(0,0,0)=\left[\begin{array}{ccc}
0 & 1 & 0 \\
\mu_{1} & -c & -\frac{\gamma}{k} \\
-\frac{k}{c} & 0 & \frac{\mu_{2}+\gamma}{c}
\end{array}\right] .
$$

Agora, para o ponto singular $B=\left(m^{-}, 0, a^{-}\right)$, onde $m^{-}$e $a^{-}$são dados em (11), obtemos

$$
J(B)=J\left(m^{-}, 0, a^{-}\right)=\left[\begin{array}{ccc}
0 & 1 & 0 \\
\mu_{1}+\frac{\gamma}{k} a^{-} & 2 \nu\left(m^{-}\right)^{q}-c & \frac{\gamma}{k}\left(m^{-}-1\right) \\
\frac{k}{c}\left(a^{-}-1\right) & 0 & \frac{k m^{-}+\mu_{2}+\gamma}{c}
\end{array}\right] .
$$

Para $J(A)$ e $J(B)$, determinaremos os autovalores $\left(\lambda_{i}\right)$ e autovetores $\left(w_{i}\right)$ correspondentes, assim, definiremos quem estará na variedade estável $\left(\operatorname{Real}\left(\lambda_{i}\right)<0\right)$ e na variedade instável $\left(\operatorname{Real}\left(\lambda_{i}\right)>0\right)$.

Resultado 1: Existência de solução na forma de onda viajante. Em [9], foram utilizados os seguintes parâmetros $\nu=8,164 \times 10^{-2}, \gamma=6,66 \times 10^{-3}, k=2,5 \times 10^{-1}, \mu_{1}=1,33 \times$ $10^{-3}, \mu_{2}=3,33 \times 10^{-4}$. Seguiremos os mesmos valores e consideraremos $q=1$ e $c=0,52$.

Descreveremos, agora, o método semi-analítico que utilizamos para estudar a existência de solução do Problema (4) que foi transformado no Sistema (8) com Condições Iniciais (9). Este método foi inspirado em [6] empenhando técnicas de sistemas dinâmicos, como a seção de Poincaré, e integração numérica no Matlab, ver a Figura 2.

- Os pontos singulares, calculados em (10) e (11), são: $A=(0,0,0)$ e $B=(0,951 ; 0 ; 0,971)$.

- Da matriz Jacobiana em cada ponto singular (Matrizes (12) e (13)), determinamos os autovalores e autovetores correspondentes. Assim, encontramos os seguintes valores

- para $J(A)$ temos os seguintes autovalores negativos $\lambda_{1}=-0,188, \lambda_{2}=-0,465 \mathrm{com}$ os respectivos autovetores $w_{1}=(0,623 ;-0,117 ; 1,490), w_{2}=(-2,826 ; 1,315 ;-2,838)$ que definem a variedade estável;

- para $J(B)$ temos os seguintes autovalores positivos $\lambda_{3}=0,063, \lambda_{4}=0,471 \mathrm{com}$ os respectivos autovetores $w_{3}=(0,997 ; 0,063 ; 0,034), w_{4}=(0,004 ; 0,002 ; 1,001)$ que definem a variedade instável.

- Criamos o Plano de Poincaré $(\pi)$ com o ponto $C=(A+B) / 2=(0,4755 ; 0 ; 0,4855)$ e o vetor normal $N=\overrightarrow{C B}=(0,4755 ; 0 ; 0,4855)$, de modo que o plano corte as variedades transversalmente.

- Obtemos a interseção entre variedades com o plano de Poincaré. A Figura 2 representa este método. 


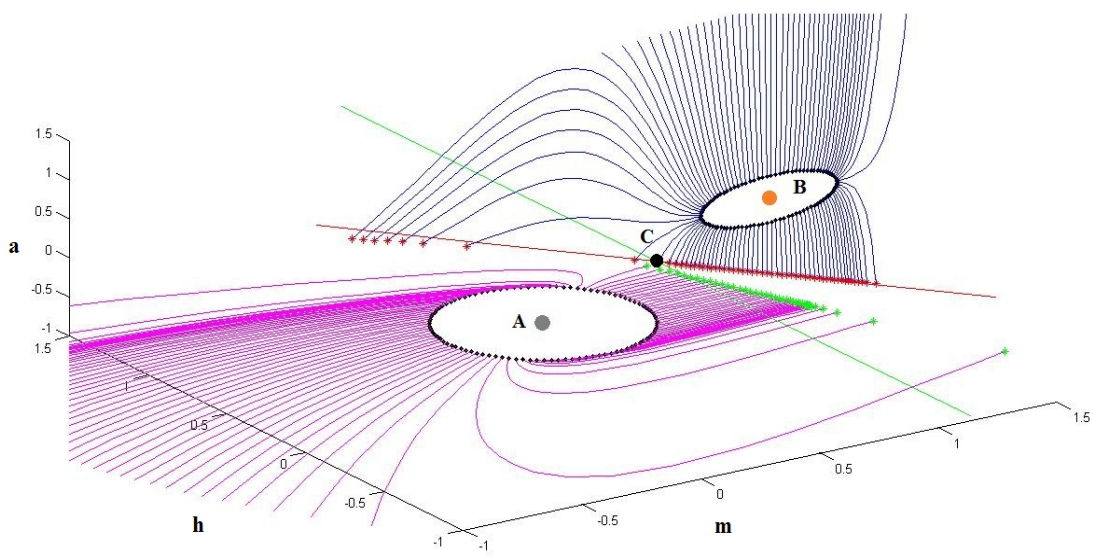

Figura 2: Os equilíbrios $A$ (cinza), $B$ (laranja) e o ponto médio $C$ (preto). As variedades estável em $A$ (linhas rosas) e a instável em $B$ (linhas azuis) interceptam o plano de Poincaré (linhas vermelha e verde) indicado pelos pontos verdes e vermelhos.

A Figura 3 representa o plano de Poincaré $(\pi)$, indicando as interseções das variedades estável em $A$ (pontos verdes) e instável em $B$ (pontos vermelhos). Encontramos uma interseção entre as curvas no plano $\pi$ (o ponto preto $P$ ), isto é, existe uma órbita que parte do equilíbrio $B$ (na variedade instável), intercepte $\pi$ e ligue ao outro equilíbrio $A$ (na variedade estável). Assim, existe uma órbita heteroclínica que ligue o equilíbrio $B$ até o equilíbrio $A$, ou seja, garantimos que existe uma solução em forma de onda viajante para o modelo.

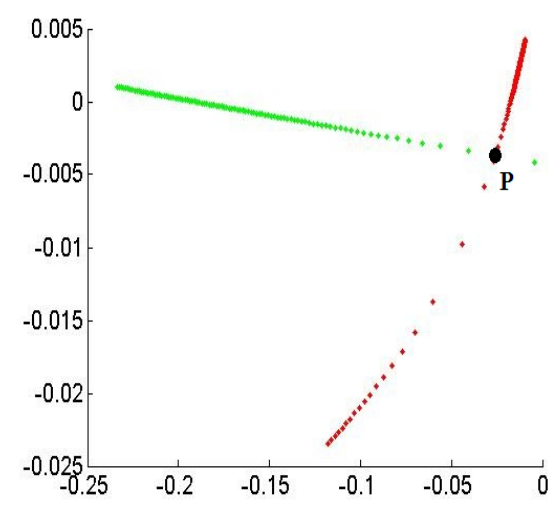

Figura 3: Plano de Poincaré da Figura 2 no qual estão indicadas as interseções com as variedades estável de $A$ (pontos verdes) e instável de $B$ (pontos vermelhos). O ponto $P$ representa a interseção entre as variedades.

Resultado 2: Solução para modelo simplificado do Sistema (4). Utilizaremos os conceitos de Leis de Conservação para mostrar outras formas de ondas e daremos uma solução para modelo simplificado do Sistema (4). Nesta análise, iremos considerar nulos os termos fontes $\left(\phi(M, A)=(\gamma / k) A(1-M)-\mu_{1} M, \psi(M, A)=k(1-A) M-\left(\mu_{2}+\gamma\right) A\right)$ e a difusão $\left(M_{x x}\right)$. Assim, obtemos o seguinte sistema na forma de Leis de Conservação $\left(M_{t}+f(M)_{x}=0\right)$ e uma EDO, isto é

$$
\left\{\begin{array}{l}
M_{t}+2 \nu M^{q} M_{x}=0 \\
A_{t}=0
\end{array}\right.
$$

Resolvemos a $1^{\text {a }}$ Equação de (14), temos o seguinte problema

$$
\left\{\begin{array}{l}
M_{t}+2 \nu M^{q} M_{x}=0 \\
M(x, 0)= \begin{cases}m_{l}, & \text { se } x<0, \\
m_{r}, & \text { se } x>0,\end{cases}
\end{array}\right.
$$


onde $m_{l}$ e $m_{r}$ são estados a esquerda e a direita, respectivamente, e o fluxo é $f^{\prime}(M)=2 \nu M^{q}$. Da $2^{\text {a }}$ Equação de (14), temos que $A_{t}(x, t)=0$, logo, $A(x, t)=g_{1}(x)+c_{1}$, onde $g_{1}$ é uma função e $c_{1}$ uma constante.

Em (15), iremos analisar dois casos, onde utilizaremos o métodos das curvas características para encontrar as soluções.

Caso 1: Seja $-\infty<m_{r}<m_{l}<\infty$ e $q>0$. Pelo método das características, obtemos

$$
\left\{\begin{array}{l}
\frac{d x}{d t}=f^{\prime}(M)=2 \nu M^{q} \\
x(0)=x_{0}
\end{array}\right.
$$

Desta forma, as curvas características são

$$
x_{s}(t)= \begin{cases}f^{\prime}\left(m_{l}\right) t+x_{0}=2 \nu m_{l}^{q} t+x_{0}, & \text { se } x_{0}<0 \\ f^{\prime}\left(m_{r}\right) t+x_{0}=2 \nu m_{r}^{q} t+x_{0}, & \text { se } x_{0}>0 .\end{cases}
$$

Pela condição Rankine-Hugoniot [4], temos que

$$
s=\frac{f\left(m_{l}\right)-f\left(m_{r}\right)}{m_{l}-m_{r}}=\frac{2 \nu}{q+1}\left(\frac{m_{l}^{q+1}-m_{r}^{q+1}}{m_{l}-m_{r}}\right)=\frac{2 \nu}{q+1}\left(\sum_{i=0}^{q} m_{l}^{q-i} m_{r}^{i}\right) .
$$

Essa é a velocidade do choque. Observamos que a $1^{\mathrm{a}}$ Equação de (15) cumpre a Condição de Entropia de Oleinik [4], pois como $m_{r}<m_{l}$ e $\nu>0$, temos que

$$
\begin{aligned}
& \left(\sum_{i=0}^{q} m_{l}^{q-i} M^{i}\right) \geq \quad\left(\sum_{i=0}^{q} m_{l}^{q-i} m_{r}^{i}\right) \quad \geq\left(\sum_{i=0}^{q} M^{q-i} m_{r}^{i}\right) \\
& \Longleftrightarrow \frac{2 \nu}{q+1}\left(\frac{M^{q+1}-m_{l}^{q+1}}{M-m_{l}}\right) \geq \frac{2 \nu}{q+1}\left(\sum_{i=0}^{q} m_{l}^{q-i} m_{r}^{i}\right) \geq \frac{2 \nu}{q+1}\left(\frac{M^{q+1}-m_{r}^{q+1}}{M-m_{r}}\right) \\
& \Longleftrightarrow \quad \frac{f(M)-f\left(m_{l}\right)}{M-m_{l}} \geq \quad s \quad \geq \frac{f(M)-f\left(m_{r}\right)}{M-m_{r}},
\end{aligned}
$$

para todo $M$ entre $m_{r}$ e $m_{l}$. Logo, a solução será em forma de choque.

Portanto, a solução do Problema (14) é

$$
\left\{\begin{array}{l}
M(x, t)= \begin{cases}m_{l}, & \text { se } x<s t, \\
m_{r}, & \text { se } x>s t\end{cases} \\
A(x, t)=g_{1}(x)+c_{1} .
\end{array}\right.
$$

Supondo que a solução do Modelo (4) se comporta como em (19), na condição $-\infty<m_{r}<m_{l}<\infty$. Assim, na solução teremos uma onda de choque seguida de uma onda viajante. A Figura 4 (a) representa esta sequência de ondas.
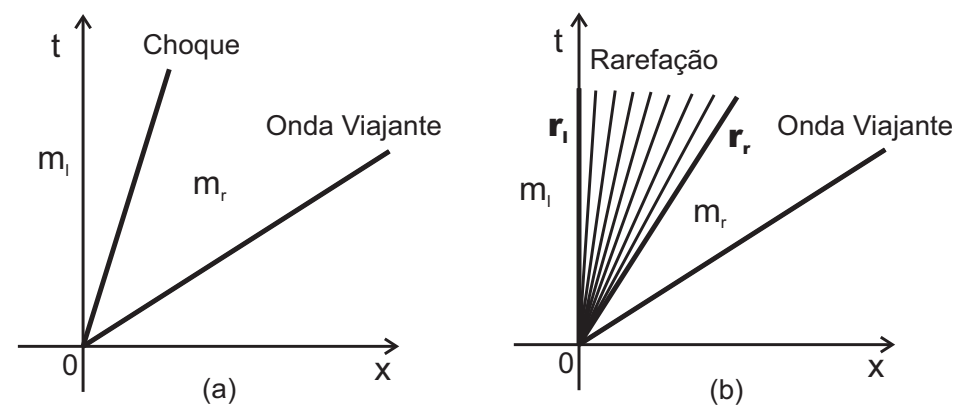

(b)

Figura 4: (a) A solução particular do Problema (4), em que $q=1, m_{l}=m^{-}$e $m_{r}=0$, é composta por uma onda de choque e por uma onda viajante. (b) A solução particular do problema (4), em que $q=1$, $m_{l}=0$ e $m_{r}=m^{-}$, é composta por uma por uma onda de rarefação e por uma onda viajante. 
Caso 2: Seja $-\infty<m_{l}<m_{r}<\infty$ e $q>0$. Buscaremos agora, a solução na forma de um onda de rarefação. As curvas características são as mesmas de (17). Sejam $M(x, t)=r(\eta)$, onde $\eta=x / t$. Logo, $M_{t}(x, t)=r^{\prime}(\eta) \frac{d \eta}{d t}$ e $M_{x}(x, t)=r^{\prime}(\eta) \frac{d \eta}{d x}$.

A solução será na forma de onda de rarefação [8], isto é

$$
r(\eta)= \begin{cases}m_{l}, & \text { se } \eta \leq f^{\prime}\left(m_{l}\right), \\ \left(f^{\prime}\right)^{-1}(\eta), & \text { se } f^{\prime}\left(m_{l}\right)<\eta<f^{\prime}\left(m_{r}\right), \\ m_{r}, & \text { se } \eta \geq f^{\prime}\left(m_{r}\right) .\end{cases}
$$

Substituindo na $1^{\text {a }}$ equação de (15) e resolvendo, obtemos $r(\eta)=\sqrt[q]{\frac{\eta}{2 \nu}}$, se $f^{\prime}\left(m_{l}\right)<\eta<f^{\prime}\left(m_{r}\right)$.

Portanto, retornando a notação inicial, onde $\eta=x / t$, e fazendo $r_{l}=f^{\prime}\left(m_{l}\right) t$ e $r_{r}=f^{\prime}\left(m_{r}\right) t$, a solução do Problema (14) para $-\infty<m_{l}<m_{r}<\infty$ é

$$
\begin{cases}M(x, t)= \begin{cases}m_{l}, & \text { se } x \leq r_{l}, \\ \sqrt[q]{\frac{x}{2 \nu t},}, & \text { se } r_{l}<x<r_{r}, \\ m_{r}, & \text { se } x \geq r_{r},\end{cases} \\ A(x, t)=g_{1}(x)+c_{1} .\end{cases}
$$

Supondo que a solução do Modelo (4) se comporta como em (20), na condição $-\infty<m_{l}<m_{r}<\infty$. Assim, teremos na sua solução uma onda de rarefação seguida de uma onda viajante. A Figura 4 (b) representa esta sequência de ondas.

Conclusões: Analisamos o modelo difusivo do Aedes aegypti baseado em [2], onde a existência de solução não foi estudada. Com este objetivo, estudamos e verificamos a existência de solução na forma de onda viajante. Estudamos também a solução particular do Problema (4), onde temos duas possibilidades de soluções dependendo das condições iniciais.

\section{Referências}

[1] C. Dufourd, Y. Dumont, Impact of environmental factors on mosquito dispersal in the prospect of sterile insect technique control, Computers $\& 3$ Mathematics with Applications, 66(9), 1695-1715, 2013.

[2] I.L. Freire, M. Torrisi, Symmetry methods in mathematical modeling of Aedes aegypti dispersal dynamics, Nonlinear Analysis: Real World Applications, 14(3), 1300-1307, 2013.

[3] S. Hay, Football fever could be a dose of dengue, Nature, 503(7477), 439-439, 2013.

[4] R.J. LeVeque, "Numerical methods for conservation laws", Birkhäuse Verlag, 1992.

[5] N.A. Maidana, H. Mo Yang, Describing the geographic spread of dengue disease by traveling waves, Mathematical biosciences, 215(1), 64-77, 2008.

[6] A.A. Mailybaev, D. Marchesin, M.H.D.S. Vera, Sensitivity analysis of stable and unstable manifolds: Theory and application, Preprint, IMPA, 2001.

[7] J. Sotomayor, "Lições de equações diferenciais ordinárias", Projecto Euclides, IMPA, 1979.

[8] C.J. van Duijn, "An introduction to conservation laws: theory and applications to multiphase flow", Lecture notes, Delft University of Technology, 2000.

[9] L.T. Takahashi, "Modelos matemáticos de epidemiologia com vetores: simulação da propagação urbana e geográfica da dengue", Tese de Doutorado, IMECC-Unicamp, 2004. 